\title{
Bifurcation theorems of Rabinowitz type for certain differential operators of the fourth order
}

\author{
by Jolanta PRZYByCin (Kraków)
}

\begin{abstract}
This paper was inspired by the works of P. H. Rabinowitz. We study nonlinear eigenvalue problems for some fourth order elliptic partial differential equations with nonlinear perturbation of Rabinowitz type. We show the existence of an unbounded continuum of nontrivial positive solutions bifurcating from $\left(\mu_{1}, 0\right)$, where $\mu_{1}$ is the first eigenvalue of the linearization about 0 of the considered problem. We also prove the related theorem for bifurcation from infinity. The results obtained are similar to those proved by Rabinowitz for second order elliptic partial differential equations ([5]-[7]). The methods used are based, in principle, on the results of [1], [5], [6].
\end{abstract}

Introduction. Let $(E,\|\|)$ be a real Banach space and let $L: E \rightarrow E$ be a compact linear operator. If there exist $\mu \in \mathbb{R}$ and $0 \neq v \in E$ such that $v=\mu L v$, then $\mu$ is said to be a real characteristic value of $L$. The set of real characteristic values of $L$ is denoted by $r(L)$.

Consider the equation

$$
u=\lambda L u+H(\lambda, \mu)
$$

where $H: \mathbb{R} \times E \rightarrow E$ is a compact operator satisfying

(0.2) $H(\lambda, u)=o(\|u\|)$ at $u=0$ uniformly on bounded $\lambda$ intervals.

By a solution of (0.1) is meant a pair $(\lambda, u) \in \mathbb{R} \times E$ satisfying (0.1). In particular, (0.1) has the line of trivial solutions $\mathcal{R}=\{(\lambda, 0) \mid \lambda \in \mathbb{R}\}$.

If (0.1) has a nontrivial solution in every neighbourhood of $(\mu, 0)$, then $(\mu, 0)$ is said to be a bifurcation point for $(0.1)$ with respect to the curve $\mathcal{R}$. It is well known that the possible bifurcation points for $(0.1)$ with respect to $\mathcal{R}$ lie on $\{(\mu, 0) \mid \mu \in r(L)\}$. Let $\mathcal{S}$ denote the closure of the set of nontrivial solutions of (0.1). A component of $\mathcal{S}$ is a maximal closed connected subset. The following was shown in [5]:

1991 Mathematics Subject Classification: 35J65, 35P30.

Key words and phrases: nonlinear partial differential equations, bifurcation in partial differential equations. 
TheOREM 0.3 (Rabinowitz). If $\mu \in r(L)$ is of odd multiplicity, then $\mathcal{S}$ contains a component $\mathcal{C}_{\mu}$ which contains $(\mu, 0)$ and is either

(i) unbounded in $\mathbb{R} \times E$, or

(ii) contains $(\widehat{\mu}, 0)$ where $\mu \neq \widehat{\mu} \in r(L)$.

Rabinowitz also proved a stronger result for a simple characteristic value $\mu$.

THEOREM 0.4. If $\mu \in r(L)$ is simple, then $\mathcal{C}_{\mu}$ can be decomposed into two subcontinua $\mathcal{C}_{\mu}^{+}, \mathcal{C}_{\mu}^{-}$such that for some neighbourhood $\mathcal{O}$ of $(\mu, 0)$, the following implication is true:

$$
(\mu, 0) \neq(\lambda, \mu) \in \mathcal{C}_{\mu}^{+}\left(\mathcal{C}_{\mu}^{-}\right) \cap \mathcal{O} \Rightarrow(\lambda, \mu)=(\lambda, \alpha v+w)
$$

where $\alpha>0(\alpha<0)$ and $|\lambda-\mu|=o(1),\|w\|=o(|\alpha|)$ at $\alpha=0$.

Rabinowitz used the above results in his later work to formulate theorems about bifurcation from infinity. Consider the equation

$$
u=\lambda L u+K(\lambda, u)
$$

where $K: \mathbb{R} \times E \rightarrow E$ is a continuous operator satisfying

(0.6) $\quad K(\lambda, u)=o(\|u\|)$ at $u=\infty$ uniformly on bounded $\lambda$ intervals.

We say $(\mu, \infty)$ is a bifurcation point for (0.5) if every neighbourhood of $(\mu, \infty)$ contains solutions of $(0.5)$, i.e. if there exists a sequence $\left(\lambda_{n}, u_{n}\right)$ of solutions of (0.5) such that $\lambda_{n} \rightarrow \mu$ and $\left\|u_{n}\right\| \rightarrow \infty$.

Let $\mathcal{T}$ denote the set of solutions of (0.5).

Theorem 0.7 (Rabinowitz). If $\mu \in r(L)$ is of odd multiplicity and $(\lambda, u) \rightarrow\|u\|^{2} K\left(\lambda, u /\|u\|^{2}\right)$ is compact, then $\mathcal{T}$ has an unbounded component $\mathcal{D}_{\mu}$ which contains $(\mu, \infty)$. Moreover, if $\Lambda \subset \mathbb{R}$ is an interval such that $\Lambda \cap r(L)=\{\mu\}$ and $\mathcal{M}$ is a neighbourhood of $(\mu, \infty)$ whose projection on $\mathbb{R}$ lies in $\Lambda$ and whose projection on $E$ is bounded away from 0 , then either

(i) $)_{\infty} \mathcal{D}_{\mu} \backslash \mathcal{M}$ is bounded in $\mathbb{R} \times E$, in which case $\mathcal{D}_{\mu} \backslash \mathcal{M}$ meets $\mathcal{R}$, or

(ii) $)_{\infty} \mathcal{D}_{\mu} \backslash \mathcal{M}$ is unbounded. If additionally $\mathcal{D}_{\mu} \backslash \mathcal{M}$ has a bounded projection on $\mathbb{R}$, then $\mathcal{D}_{\mu} \backslash \mathcal{M}$ contains $(\widehat{\mu}, \infty)$ where $\mu \neq \widehat{\mu} \in r(L)$.

Theorem 0.8 (Rabinowitz). If $\mu \in r(L)$ is simple, then $\mathcal{D}_{\mu}$ can be decomposed into two subcontinua $\mathcal{D}_{\mu}^{+}, \mathcal{D}_{\mu}^{-}$and there exists a neighbourhood $\mathcal{O} \subset \mathcal{M}$ such that the following implication is true:

$$
(\mu, \infty) \neq(\lambda, u) \in \mathcal{D}_{\mu}^{+}\left(\mathcal{D}_{\mu}^{-}\right) \cap \mathcal{O} \Rightarrow(\lambda, u)=(\lambda, \alpha v+w)
$$

where $\alpha>0(\alpha<0)$ and $|\lambda-\mu|=o(1),\|w\|=o(|\alpha|)$ at $|\alpha|=\infty$. 
1. Existence of positive solutions for nonlinear elliptic partial differential equations of the fourth order. Let $\Omega$ be a bounded domain in $\mathbb{R}^{n}$ with smooth boundary $\partial \Omega$ and let $\mathcal{N}$ be a differential operator of the form $\mathcal{N}=\mathcal{N}_{1} \circ \mathcal{N}_{0}$, where

$$
\mathcal{N}_{k} u=-\sum_{i, j=1}^{n} \frac{\partial}{\partial x_{i}}\left(a_{i j}^{k} \frac{\partial u}{\partial x_{j}}\right)+q^{k} u, \quad k=0,1 .
$$

We assume that $a_{i j}^{k}=a_{j i}^{k} \in C^{3-2 k}(\bar{\Omega}), 0 \leq q^{k} \in C^{2-2 k}(\bar{\Omega})$ and the quadratic forms $\sum_{i, j=1}^{n} a_{i j}^{k} \xi_{i} \xi_{j}$ are positive definite in $\bar{\Omega}$.

Consider the nonlinear equation

$$
\mathcal{N} u=\lambda(u+h(\cdot, u)) \quad \text { in } \Omega
$$

together with the boundary conditions

$$
u=\mathcal{N}_{0} u=0 \quad \text { on } \partial \Omega .
$$

Assume that

(1.2) $\quad h$ is continuous on $\bar{\Omega} \times \mathbb{R}$ and $h(x, \xi)=o(|\xi|)$ at $\xi=0$ uniformly in $x \in \bar{\Omega}$.

Let $E=C^{3}(\bar{\Omega}) \cap$ (B.C.). $E$ is a Banach space equipped with its usual norm $\|u\|_{3}=\sum_{|\alpha| \leq 3} \max \left|D^{\alpha} u(x)\right|$.

If $h=0$ then the linear problem $\mathcal{N} v=\mu v$ in $\Omega$ together with the boundary conditions (B.C.) has a smallest positive eigenvalue $\mu_{1}$, which is simple, and such that a corresponding eigenfunction $v_{1}$ is positive in $\Omega([1])$. By requiring $\left\|v_{1}\right\|_{3}=1, v_{1}$ is uniquely determined. Using the strong maximum principle and the positivity of $\mathcal{N}_{1}^{-1}$ it is easy to show that $v_{1} \in P^{+}=\{u \in E \mid u>0$ in $\Omega, \partial u / \partial \nu<0$ on $\partial \Omega\}$, where $\partial u / \partial \nu$ denotes the outward normal derivative of $u$. The sets $P^{+}, P^{-}=-P^{+}, P=P^{+} \cup P^{-}$ are open in $E$.

We will convert (1.1) into an equivalent operator equation in $\mathbb{R} \times E$ of the form $(0.1)$.

Let $(\lambda, u) \in \mathbb{R} \times E$ and consider the linear equation

$$
\mathcal{N} v=\lambda(u+h(\cdot, u)) \quad \text { in } \Omega
$$

together with the boundary conditions $v=\mathcal{N}_{0} v=0$ on $\partial \Omega$. We can write the above problem in the form

$$
\begin{array}{ll}
\mathcal{N}_{1} w=\lambda(u+h(\cdot, u)) & \text { in } \Omega, w=0 \text { on } \partial \Omega, \\
\mathcal{N}_{0} v=w & \text { in } \Omega, v=0 \text { on } \partial \Omega .
\end{array}
$$

For $p>1$, let $W_{0}^{m, p}(\Omega)$ denote the closure of the $m$ times continuously 
differentiable functions in $\bar{\Omega}$ which satisfy (B.C.) on $\partial \Omega$ in the norm

$$
|u|_{m, p}=\left(\sum_{|\alpha| \leq m} \int_{\Omega}\left|D^{\alpha} u(x)\right|^{p} d x\right)^{1 / p}
$$

and let $|\cdot|_{0, p}=|\cdot|_{p}$. Clearly $W_{0}^{0, p}(\Omega)=L^{p}(\Omega)$.

Notice that the right-hand side of the considered equation as a continuous function on $\bar{\Omega}$ lies in $L^{p}(\Omega)$ for all $p>1$. The $L^{p}$ theory for uniformly elliptic partial differential equations implies the existence of a unique $w \in W_{0}^{2, p}(\Omega)$ satisfying

$$
|w|_{2, p} \leq \text { const }|\lambda(u+h(\cdot, u))|_{p} .
$$

Arguing as above once again we obtain a unique solution $v=G(\lambda, u) \in$ $W_{0}^{4, p}(\Omega)$ of (1.3) satisfying

$$
|v|_{4, p} \leq \text { const }|w|_{2, p} \leq \text { const }|\lambda(u+h(\cdot, u))|_{p} .
$$

For $p>n, W_{0}^{4, p}$ is compactly embedded in $E$ ([3]). Thus choosing any $p>n$ we obtain the compactness of $G: \mathbb{R} \times E \rightarrow E$. Any solution of (1.1) satisfies $u=G(\lambda, u)$ and conversely.

Let $w=L u \in W_{0}^{4, p}(\Omega)$ denote the unique solution of $\mathcal{N} w=u$ in $\Omega$ together with the boundary conditions $w=\mathcal{N}_{0} w=0$ on $\partial \Omega$. Then as above $L: E \rightarrow E$ is a compact linear operator. Define $H(\lambda, u)=G(\lambda, u)-\lambda L u$. We have shown that equation (1.1) is equivalent to $u=\lambda L u+H(\lambda, u)$. We must verify that $H$ satisfies $(0.2)$.

Fix $\varepsilon>0$ and let $\Lambda \subset \mathbb{R}$ be a bounded interval. By the $L^{p}$ estimates,

$$
|H(\lambda, u)|_{4, p} \leq \text { const }|h(\cdot, u)|_{p} .
$$

Moreover,

$$
|h(\cdot, u)|_{p} \leq \text { const } \max _{x \in \bar{\Omega}}|h(x, u(x))| .
$$

By the assumption (1.2) we have

$$
\exists \delta>0 \quad|\xi|<\delta \Rightarrow|h(x, \xi)|<\varepsilon|\xi| .
$$

Hence, for $(\lambda, u) \in \Lambda \times \widetilde{S}=\left\{(\lambda, u) \in \Lambda \times E \mid\|u\|_{3}<\delta\right\}$ we obtain the estimate

$$
|H(\lambda, u)|_{4, p} \leq \text { const } \max _{x \in \bar{\Omega}}|h(x, u(x))| \leq \text { const } \varepsilon\|u\|_{3} .
$$

Therefore $H$ satisfies (0.2).

Let $\mathcal{S}$ denote the closure of the set of nontrivial solutions of (1.1) in $\mathbb{R} \times E$. The hypotheses of Theorem 0.3 are satisfied and so there exists a component $\mathcal{C}_{1}$ of $\mathcal{S}$ containing $\left(\mu_{1}, 0\right)$ and satisfying the alternatives of Rabinowitz. Actually only the first alternative is possible as will be shown below. We first prove two important lemmas. 
LEMmA 1.4. (a) There exists a neighbourhood $U_{1}$ of $\left(\mu_{1}, 0\right)$ such that $(\lambda, u) \in U_{1} \cap \mathcal{S}$ and $u \neq \equiv$ implies $u \in P$.

(b) If $\mu_{1} \neq \widehat{\mu} \in r(L)$ there exists a neighbourhood $\widehat{U}$ of $(\widehat{\mu}, 0)$ such that $(\lambda, u) \in \widehat{U} \cap \mathcal{S}$ and $u \not \equiv 0$ implies $u \notin P$.

Proof. (a) If not, there exists a sequence $\left(\lambda_{n}, u_{n}\right) \in \mathcal{S}$ such that $0 \not \equiv$ $u_{n} \notin P,\left(\lambda_{n}, u_{n}\right) \rightarrow\left(\mu_{1}, 0\right)$ in $\mathbb{R} \times E$ and

$$
\frac{u_{n}}{\left\|u_{n}\right\|_{3}}=\lambda_{n} L \frac{u_{n}}{\left\|u_{n}\right\|_{3}}+\frac{H\left(\lambda_{n}, u_{n}\right)}{\left\|u_{n}\right\|_{3}} .
$$

Since $L$ is compact, a subsequence of $L u_{n} /\left\|u_{n}\right\|_{3}$ converges. Hence the left-hand side of the above equation has a convergent subsequence $u_{n_{p}} /\left\|u_{n_{p}}\right\|_{3} \rightarrow w$ with $\|w\|_{3}=1$ and $w=\mu_{1} L w$. Consequently, $w=$ $\pm v_{1} \in P$. Since $P$ is open, $u_{n_{p}} \in P$ for $p$ sufficiently large, contrary to the assumption.

(b) We proceed analogously. Assume that there exists a sequence $\left(\lambda_{n}, u_{n}\right) \in \mathbb{R} \times P$ such that $\left(\lambda_{n}, u_{n}\right) \rightarrow(\widehat{\mu}, 0)$ in $\mathbb{R} \times E$. Then $\bar{P} \ni w=$ $\lim _{n \rightarrow \infty} u_{n} /\left\|u_{n}\right\|_{3}$ satisfies $w=\mu L w$. If $w \in \partial P^{+}$, then either $w$ has a zero in $\Omega$ or $\partial w / \partial \nu=0$ on $\partial \Omega$. Using the positivity of $\mathcal{N}_{1}^{-1}$ and a maximum principle argument we see that $w= \pm v_{1}$, which contradicts the choice of $\widehat{\mu} \neq \mu_{1}$.

Lemma 1.5. If $\left(\lambda_{n}, u_{n}\right) \in\left(\mathbb{R}^{+} \times P\right) \cap \mathcal{S}$ is a sequence converging to $(\lambda, u) \in \mathcal{C}_{1} \backslash\left\{\left(\mu_{1}, 0\right)\right\}$, then $(\lambda, u) \in \mathbb{R}^{+} \times P$.

Pro of. We first prove that $\lambda>0$. Indeed, otherwise there would exist a pair $(0, u) \in \mathcal{C}_{1}$ satisfying the assumptions of the lemma. This would mean that $u$ is a unique solution of (1.1) with the right-hand side equal to 0 . Hence $(0, u)=(0,0)$ would be a bifurcation point of $(1.1)$, which is impossible. It remains to show that $u \in P$. Assume, to the contrary, $u \in \partial P^{+}$. The definition of $P^{+}$implies that either

(i) there exists $\xi \in \Omega$ such that $u(\xi)=0$, or

(ii) there exists $\eta \in \partial \Omega$ such that $(\partial u / \partial \nu)(\eta)=0$.

Suppose that (i) occurs. The condition (1.2) guarantees the existence of a neighbourhood $U$ of $\xi$ such that $|h(x, u(x))| \leq u(x) / 2$ for $x \in U$. Hence

$$
\begin{array}{clrl}
\mathcal{N} u(x) & \geq \lambda u(x) / 2 & & \text { for } x \in U, \\
u(x) \geq 0 & & \text { for } x \in \partial U .
\end{array}
$$

Then applying the positivity of $\mathcal{N}_{1}^{-1}$ we obtain

$$
\begin{aligned}
\mathcal{N}_{0} u(x) \geq 0 & \text { for } x \in U, \\
u(x) \geq 0 & \text { for } x \in \partial U .
\end{aligned}
$$


Since $u$ has a local minimum at $\xi$, the elliptic maximum principle implies $u \equiv 0$ in $U$. By Lemma 1.4 we obtain $(\lambda, u)=\left(\mu_{1}, 0\right)$, which is impossible.

If (ii) occurs the argument is analogous. There exists a neighbourhood $U$ of $\eta \in \partial \Omega$ in which (1.6) holds. Using the strong version of the elliptic maximum principle we obtain $u \equiv 0$ in $U$. Then $(\lambda, u)=\left(\mu_{1}, 0\right)$. The proof is complete.

Actually we can prove a global bifurcation theorem for (1.1).

THEOREM 1.7. There exists an unbounded component of $\mathcal{S}, \mathcal{C}_{1}$, bifurcating from $\left(\mu_{1}, 0\right)$ and contained in $\left(\mathbb{R}^{+} \times P\right) \cup\left\{\left(\mu_{1}, 0\right)\right\}$.

Proof. It suffices to show that $\mathcal{C}_{1} \subset\left(\mathbb{R}^{+} \times P\right) \cup\left\{\left(\mu_{1}, 0\right)\right\}$. Indeed, otherwise the connectedness of $\mathcal{C}_{1}$ and Lemma 1.4 imply that there exists a pair $(\lambda, u) \in \mathbb{R}^{+} \times \partial P$ satisfying the assumptions of Lemma 1.5. Then $(\lambda, u) \in \mathbb{R}^{+} \times P$, a contradiction.

Remark. By using Theorem 0.4 we can decompose $\mathcal{C}_{1}$ into two unbounded subcontinua $\mathcal{C}_{1}^{+}, \mathcal{C}_{1}^{-}$such that $\mathcal{C}_{1}^{ \pm} \subset\left(\mathbb{R}^{+} \times P\right) \cup\left\{\left(\mu_{1}, 0\right)\right\}$.

2. Bifurcation from infinity for a nonlinear elliptic eigenvalue problem of the fourth order. Consider the nonlinear equation

$$
\mathcal{N} u \equiv \mathcal{N}_{1} \circ \mathcal{N}_{0} u=\lambda u+k(\cdot, u, \lambda) \quad \text { in } \Omega
$$

together with the boundary conditions (B.C.). All the assumptions and notations from Section 1 are still valid. Assume additionally that the righthand side of (2.1) is continuous on $\bar{\Omega} \times \mathbb{R}^{2}$ and

$$
\begin{aligned}
& k(x, \xi, \lambda)=o(|\xi|) \text { at } \xi=\infty \text { uniformly in } x \in \bar{\Omega} \text { and } \lambda \in \Lambda \text {, for any } \\
& \text { bounded interval } \Lambda \subset \mathbb{R} \text {. }
\end{aligned}
$$

Similarly to Section 1 we can convert (2.1) into an equivalent operator equation in $\mathbb{R} \times E=\mathbb{R} \times\left(C^{3}(\bar{\Omega}) \cap\right.$ (B.C. $\left.)\right)$,

$$
u=\lambda L u+K(\lambda, u) .
$$

Let $\mathcal{T}$ denote the set of solutions of $(2.1)$ in $\mathbb{R} \times E$.

THEOREM 2.4. There exists a component $\mathcal{D}_{1}$ of $\mathcal{T}$ which contains $\left(\mu_{1}, \infty\right)$ and satisfies the conclusions of Theorems 0.7 and 0.8 . Moreover, there exists a neighbourhood $\mathcal{O}$ of $\left(\mu_{1}, \infty\right)$ such that $\mathcal{D}_{1} \cap \mathcal{O} \subset\left(\mathbb{R}^{+} \times P\right) \cup$ $\left\{\left(\mu_{1}, \infty\right)\right\}$.

Proof. We will show that the equation (2.3) is of the form (0.5) and that the operator $\widehat{K}:(\lambda, u) \rightarrow\|u\|_{3}^{2} K\left(\lambda, u /\|u\|_{3}^{2}\right)$ is compact. Clearly $K$ is continuous on $\mathbb{R} \times E$, and $L$ is compact and linear. To show that $K$ satisfies (0.6) let $\Lambda \subset \mathbb{R}$ be a bounded interval and fix $\varepsilon>0$. By the $L^{p}$ 
estimates,

$$
|K(\lambda, u)|_{4, p} \leq \mathrm{const}|k(\cdot, u, \lambda)|_{p} \leq \mathrm{const} \max _{x \in \bar{\Omega}}|k(x, u(x), \lambda)| .
$$

The assumption (2.2) implies

$$
\exists \delta>0 \quad|\xi|>\delta \Rightarrow|k(x, \xi, \lambda)|<\varepsilon|\xi| .
$$

Moreover, the continuity of $k$ yields $|k(x, \xi, \lambda)| \leq M$ for $|\xi| \leq \delta$.

Choose $\delta_{1}$ so large that $M / \delta_{1}<\varepsilon$ and define $S=\left\{u \in E \mid\|u\|_{3} \geq \delta_{1}\right\}$. Hence for $(\lambda, u) \in \Lambda \times S$ we obtain

$$
\begin{aligned}
\max _{x \in \bar{\Omega}}|k(x, u(x), \lambda)| & \leq \max _{\lambda \in \Lambda,|u(x)| \leq \delta}|k(x, u(x), \lambda)|+\max _{\lambda \in \Lambda,|u(x)|>\delta}|k(x, u(x), \lambda)| \\
& \leq M+\varepsilon\|u\|_{3}<2 \varepsilon\|u\|_{3} .
\end{aligned}
$$

Now, (2.5) shows that

$$
|K(\lambda, u)|_{4, p}<2 \text { const } \varepsilon\|u\|_{3},
$$

and by the Sobolev inequality we finally have $\|K(\lambda, u)\|_{3}<\varepsilon \widetilde{c}\|u\|_{3}$ for $(\lambda, u) \in \Lambda \times S$. Hence $K$ satisfies (0.6).

To verify that $\widehat{K}$ is compact, notice that $\widehat{K}\left(\left\{(\lambda, u) \in \Lambda \times E \mid \varrho_{1} \leq\right.\right.$ $\left.\left.\|u\|_{3} \leq \varrho_{2}\right\}\right)$ is relatively compact for any $0<\varrho_{1} \leq \varrho_{2}<\infty$. Thus we need only prove that $\widehat{K}\left(\Lambda \times \bar{B}_{1 / \delta_{1}}\right)$ is relatively compact in $E$, where $\bar{B}_{1 / \delta_{1}}=$ $\left\{u \in E \mid\|u\|_{3} \leq 1 / \delta_{1}\right\}$.

From (2.6) we obtain the boundedness of $\widehat{K}\left(\Lambda \times \bar{B}_{1 / \delta_{1}}\right)$. In fact,

$|\widehat{K}(\lambda, u)|_{4, p}=\|u\|_{3}^{2}\left|K\left(\lambda, u /\|u\|_{3}^{2}\right)\right|_{4, p}<\operatorname{const} \varepsilon / \delta_{1} \quad$ for $(\lambda, u) \in \Lambda \times \bar{B}_{1 / \delta_{1}}$.

Since $E$ is compactly embedded in $W_{0}^{4, p}(\Omega)$ it follows that $\widehat{K}\left(\Lambda \times \bar{B}_{1 / \delta_{1}}\right)$ is relatively compact. So we can apply Theorems 0.7 and 0.8 . It remains to show that for $\mathcal{O}$ sufficiently small,

$$
\left(\mu_{1}, \infty\right) \neq(\lambda, u) \in \mathcal{D}_{1}^{+}\left(\mathcal{D}_{1}^{-}\right) \cap \mathcal{O} \Rightarrow u \in P^{+}\left(P^{-}\right) .
$$

By Theorem 0.7 we know that

$$
\left(\mu_{1}, \infty\right) \neq(\lambda, u) \in \mathcal{O} \cap \mathcal{D}_{1}^{+}\left(\mathcal{D}_{1}^{-}\right) \Rightarrow(\lambda, u)=\left(\lambda, \alpha v_{1}+w\right)
$$

where $\alpha>0(\alpha<0)$ and $\|w\|_{3}=o(|\alpha|)$ at $|\alpha|=\infty$. Since $P^{+}\left(P^{-}\right)$is open, $v_{1}+w / \alpha \in P^{+}\left(P^{-}\right)$for $\alpha$ near $\infty$ and therefore $u=\alpha v_{1}+w \in P^{+}\left(P^{-}\right)$. Thus the proof is complete.

COROLlary. If we assume additionally that $k(x, \xi, \lambda)=\lambda h(x, \xi)$, where $h$ satisfies $(1.2)$, then $\mathcal{D}_{1}^{ \pm} \backslash \mathcal{O}$ contains a subcontinuum lying in $\mathbb{R}^{+} \times P^{ \pm}$ which is either unbounded or intersects the line $\mathcal{R}$ of trivial solutions at $\left(\mu_{1}, 0\right)$.

Proof. We know that $\mathcal{D}_{1}^{ \pm} \cap \mathcal{O} \subset\left(\mathbb{R}^{+} \times P\right) \cup\left\{\left(\mu_{1}, \infty\right)\right\}$. Let $\mathcal{E}_{1}^{+}$denote the maximal subcontinuum of $\mathcal{D}_{1}^{+}$contained in $\mathbb{R}^{+} \times P^{+}$. Suppose $\mathcal{E}_{1}^{+} \backslash \mathcal{O}$ is 
bounded. Then there exists a pair $(\lambda, u) \in \partial \mathcal{E}_{1}^{+} \cap\left(\mathbb{R}^{+} \times P^{+}\right)$. By Lemma 1.5, $u \equiv 0$. Hence $\left(\mathcal{E}_{1}^{+} \backslash \mathcal{O}\right) \cap \mathcal{R} \neq \emptyset$. Applying Lemma 1.4 we know that only $\left(\mu_{1}, 0\right)$ can be the limit of solutions $(\lambda, u) \in \mathbb{R}^{+} \times P^{+}$. Hence finally $\left(\mathcal{E}_{1}^{+} \backslash \mathcal{O}\right) \cap \mathcal{R}=\left\{\left(\mu_{1}, 0\right)\right\}$, which was to be shown.

Remark. The methods used in this paper cannot be applied to the operator $\mathcal{N}$ of the form $\mathcal{N}=\mathcal{N}_{m} \circ \mathcal{N}_{m-1} \circ \ldots \circ \mathcal{N}_{1} \circ \mathcal{N}_{0}$ where $m>1$ because the operator $\mathcal{N}_{1}^{-1} \circ \mathcal{N}_{2}^{-1} \circ \ldots \circ \mathcal{N}_{m}^{-1}$ is not necessarily positive, and the positivity is essential for our methods.

\section{References}

[1] J. Bochenek, On some properties of eigenvalues and eigenfunctions of certain differential equations of the fourth order, Ann. Polon. Math. 24 (1971), 113-119.

[2] -, Positive solution of asymptotically linear elliptic eigenvalue problems for certain differential equations of the fourth order, ibid. 45 (1985), 231-236.

[3] L. Nirenberg, Topics in Nonlinear Functional Analysis, Courant Institute, New York 1974.

[4] J. Przybycin, Some applications of bifurcation theory to ordinary differential equations of the fourth order, Ann. Polon. Math. 53 (1991), 153-160.

[5] P. H. Rabinowitz, Some aspects of nonlinear eigenvalue problems, Rocky Mountain J. Math. 3 (1973), 161-202.

[6] -, On bifurcation from infinity, J. Differential Equations 14 (1973), 462-475.

[7] - Some global results for nonlinear eigenvalue problems, J. Funct. Anal. 7 (1971), $487-513$.

INSTITUTE OF MATHEMATICS

ACADEMY OF MINING AND METALLURGY

MICKIEWICZA 30

30-059 KRAKÓW, POLAND 